\title{
Faculty Survey
}

\section{9}

Department of Medicine (DoM) Faculty Survey 2019

CONSENT TO PARTICIPATE

You may start and return to this survey over multiple sittings, at your convenience. Your answers on a completed page are preserved, and when you next click on the survey link, you will be taken to the next section following your last completed page. You can change your answers on any survey page(s) until you click 'submit' at the end.

\section{* 1. Confirmation}

I wish to continue and complete the survey to help inform the Department of Medicine (DoM) on issues related to Career/Work Place Satisfaction.

I wish to opt-out of this survey. 


\section{Faculty Survey}

Department of Medicine (DoM) Faculty Survey 2019

INSTRUCTIONS \& NOTES

We anticipate that this iteration of the Faculty Survey will take approximately 15 minutes to complete.

When completing this survey, if you are cross-appointed to two or more divisions, we ask that you answer questions from the perspective of the SINGLE division with which you most identify (i.e. the division in which you spend more clinical time, have your most meaningful scholarly activity, and conduct most of your mentoring).

Abbreviations / Clarification of terms:

- PIC: Physician-in-Chief, the head of the hospital department of medicine

- DoM: Department of Medicine

- DDD: Departmental Division Director (Citywide), the head of the citywide division to which you belong

- Hospital Division Head (Local), the head of the hospital division to which you belong

- CIHR: Canadian Institutes of Health Research

- CFA: Clinical Faculty Advocate (See: http://www.deptmedicine.utoronto.ca/clinical-facultyadvocate) 


\section{Faculty Survey}

\section{9}

Department of Medicine (DoM) Faculty Survey 2019

SECTION 1: WORK-LIFE INTEGRATION \& WELLNESS

Definition of Work-Life Integration.

The following questions seek to assess your career satisfaction as a member of the DoM and is based on the Stanford Professional Fulfillment Index which is a validated wellness screening tool (Trockel et al., 2017).

2. How true do you think the following statements are about you at work during the past two weeks?

Select ONE response for each option provided.

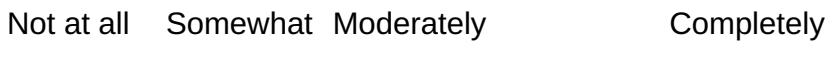

I feel happy at work

I feel worthwhile at work

My work is satisfying to me

I feel in control when dealing with difficult problems at work

My work is meaningful to me

I'm contributing professionally (eg. Patient care, teaching,

research, and leadership) in the ways I value most

3. During the past two representative (clinical / scholarly) weeks I have felt...

Select ONE response for each option provided.

Not at all Very little Moderately A Lot Extremely

A sense of dread when I think about work I have to do

Physically exhausted at work

Lacking in enthusiasm at work

Emotionally exhausted at work 
4. During the past two representative (clinical / scholarly) weeks, my job has contributed to me feeling...

Select ONE response for each option provided.

Not at all Very little Moderately A Lot Extremely

Less empathetic with my patients

Less empathetic with my colleagues

Less sensitive to others' feelings / emotions

Less interested in talking with my patients

Less connected with my patients

Less connected with my colleagues 


\section{Faculty Survey}

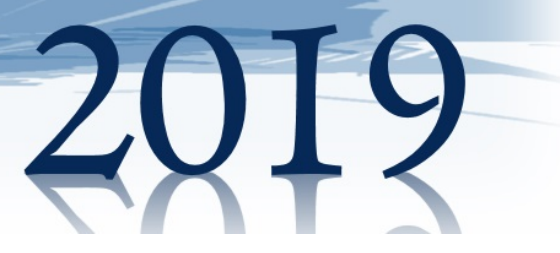

Department of Medicine (DoM) Faculty Survey 2019

\section{SECTION 2: CIVILITY / PROFESSIONALISM}

The following questions address issues related to equity, diversity and professionalism.

Click here for Glossary reference.

5. Please rate your satisfaction with the efforts made by... to create a collegial and supportive environment that promotes inclusivity.

Select ONE response for each option provided. Note: This question is based on your satisfaction with the efforts made, and not your overall satisfaction with the environment itself.

Not Strongly Somewhat Somewhat Strongly Applicable Dissatisfied Dissatisfied Neutral Satisfied Satisfied

University DoM

University Division

Your Primary Hospital DoM

Your Primary Hospital Division

6. The people I work with at (my) ... interact with me in a respectful and civil manner. Select ONE response for each option provided.

$\begin{array}{cccc}\text { Not Strongly Somewhat } & \text { Somewhat Strongly } \\ \text { Applicable Disagree Disagree } & \text { Neutral } & \text { Agree Agree }\end{array}$

University DoM

University Division

My Primary Hospital DoM

My Primary Hospital Division

7. I am aware of policies and procedures in place at ... to address unprofessionalism (for example, issues of disrespect, abuse, bullying, microaggression, and discrimination).

Select ONE response for each option provided.

$\begin{array}{lcccc}\text { N/A (Not } \\ \text { University DoM } & \text { No } & \text { Uncertain } & \text { Yes Applicable) } \\ \text { My Primary Hospital } & & & \end{array}$


8. Please rate your confidence that you cantake action to address unprofessionalism at ... without concern for reprisal.

Select ONE response for each option provided.

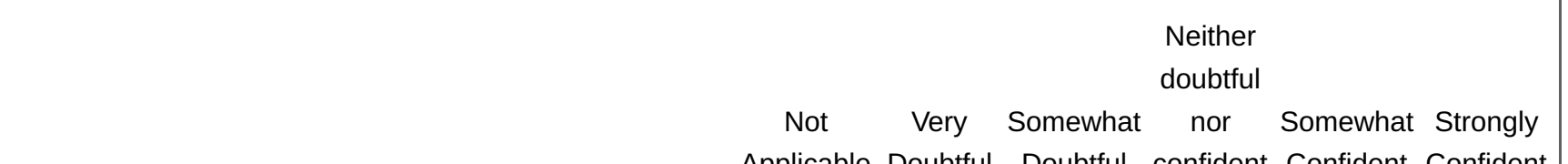

University DoM

University Division

My Primary Hospital DoM

My Primary Hospital Division

* 9. Have you witnessed what you perceive to be "unprofessionalism" (for example, issues of disrespect, abuse, bullying, microaggression, and discrimination) by faculty members, towards others (for example trainees, other academic faculty within the department or otherwise, interprofessional colleagues) at your hospital or at the university, within the last two years? Select ONE response only.

Applicable Doubtful Doubtful confident Confident Confident

$\bigcirc$ Yes
No

* 10. Have you personally experienced what you perceive to be "unprofessionalism" (for example, issues of disrespect, abuse, bullying, microaggression, and discrimination) in the Department (at your hospital or at the university) within the last two years?

Select ONE response only.

Yes

No

11. Please feel free to provide any comments to elaborate on your answers to any of the questions on this survey page. 


\section{Faculty Survey}

\section{9}

Department of Medicine (DoM) Faculty Survey 2019

SECTION 2: CIVILITY / PROFESSIONALISM (PERSONAL EXPERIENCE)

The following questions refer to your collective experiences with unprofessionalism.

12. What types or form(s) of unprofessionalism did these events (collectively) include? Select ALL responses that apply.
Disrespect
Abuse
Bullying
Microaggression
Discrimination
Other (please specify)

13. Did you perceive any of these events to be attributed to your identification with a specific minority group?

Select ALL responses that apply.
No
Gender
Race/Ethnicity
Sexual Orientation
$\square$ Other (please specify)

14. What was the impact of these events on you personally or professionally? Please comment. If you want to access resources for support or additional guidance, please click here.

* 15. Did you take action/report any of the events to a direct supervisor at the time? Select ONE response only.
Yes
No 
16. Comments in regards to taking action/reporting: 


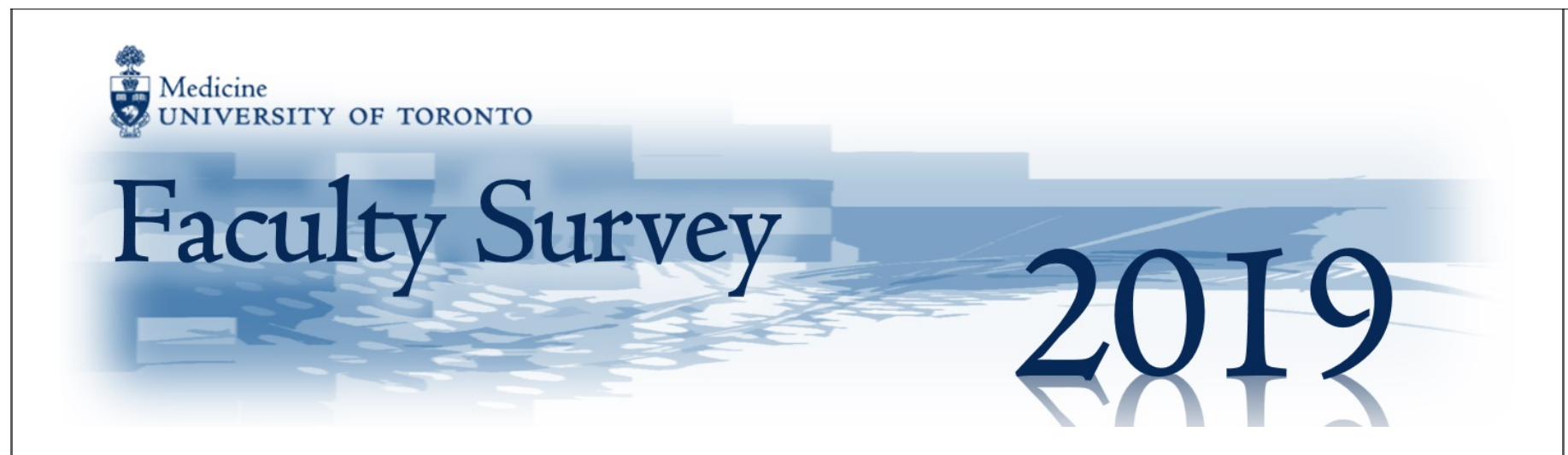

Department of Medicine (DoM) Faculty Survey 2019

SECTION 2: CIVILITY / PROFESSIONALISM (REPORTING)

17. Did you experience any reprisal after you reported any of the events? Select ONE response only.

$\bigcirc$ Yes
No

Comments

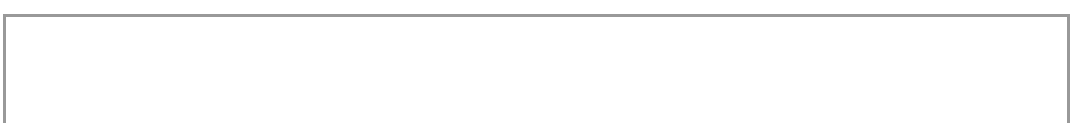

18. To your knowledge, was there any action taken as a result of your complaint (e.g., investigation)?

Select ONE response only.
I don't know
Yes
No 


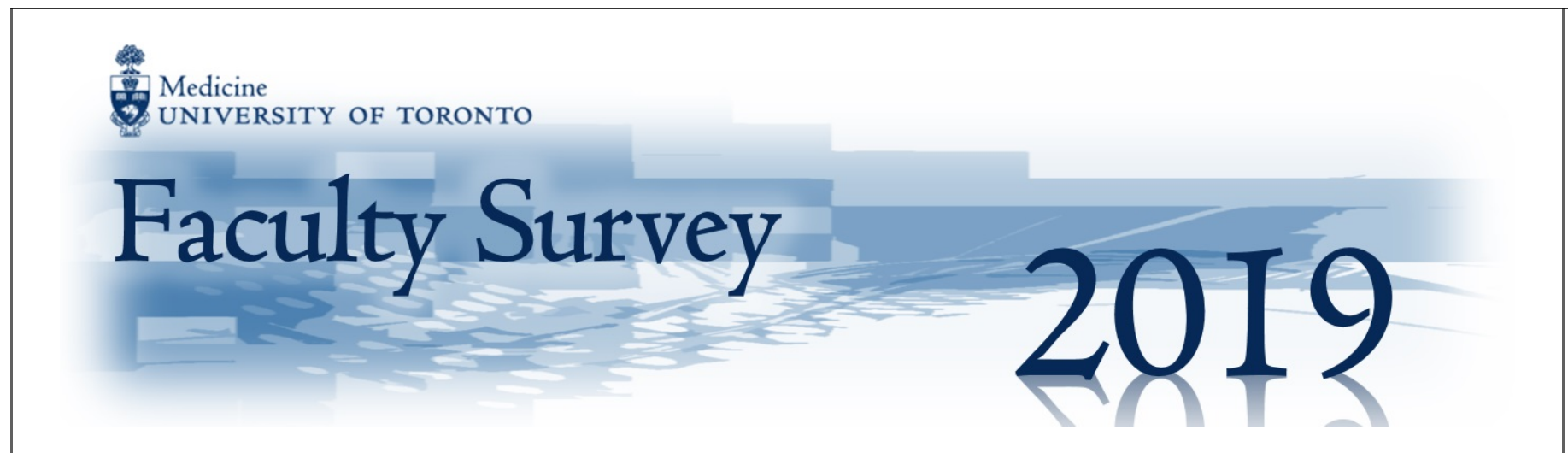

Department of Medicine (DoM) Faculty Survey 2019

SECTION 2: CIVILITY / PROFESSIONALISM (Cont'd)

19. Have you had any formal unconscious bias training (e.g., Harvard Implicit Association Test, or other similar training) within the last two years?

Select ONE response only.

Yes

No 


\section{Faculty Survey}

\section{9}

Department of Medicine (DoM) Faculty Survey 2019

SECTION 3: WORK STRUCTURE

The following questions seek to understand how work structures, including schedules, remuneration and benefits, leaves of absence, meetings, and electronic tools, affect the experiences and wellness of faculty members.

20. Are you interested in reducing your clinical workload to help with yourwork-life integration? Select ONE response only.
Yes
No
Not sure 


\section{Faculty Survey}

\section{9}

Department of Medicine (DoM) Faculty Survey 2019

SECTION 3: WORK STRUCTURE (Cont'd)

21. Please rate your level of interest in the following strategies to enable you to reduce your clinical workload.

Please select ONE response for each option provided.

Definitely

not Slightly Moderately Very Extremely Interested Interested Interested Interested Interested

Shift Work (e.g., defined hours of work over days / evenings

/ weekends / nights)

Part-time work

Work Sharing

(2 individuals sharing a full-time position)

Reducing clinical hours by delegating part of the clinical workload to advanced practice providers (e.g., Nurse practitioners or Physician assistants)

Duty hour restrictions (e.g. having different staff provide day versus night coverage)

Alternative funding models to reduce dependence on fee for service income (e.g. mixed stipend + fee for service)

Expanded faculty numbers

\section{Comments}


22. Please indicate your willingness to accept the following

compromises that may need to be undertaken to help with your work-

life integration.

Select ONE response for each option provided.

Willing to accept Not willing to accept

Reduction in total income

Reduction in clinical income

Reduction in clinical hours

Becoming a salaried employee

Reduction in continuity (with patients)

Reduction in continuity (with trainees)

Delayed CFAR [4] or promotion

Comments

[4] CFAR = continuing faculty appointment review (3-year review). 


\section{Faculty Survey}

\section{9}

Department of Medicine (DoM) Faculty Survey 2019

SECTION 3: WORK STRUCTURE (Cont'd)

23. Would you be interested in being remunerated with a "fixed salary" (akin to working as an employee)?

Select ONE response only.

Yes

No

Not sure

It depends, please provide a comment

leave?

Select ONE response only.

$\bigcirc$ Yes
No

Comments

(2)

25. Please indicate if you think the following types of leave should be formally accommodated by your hospital DoM (which may require contributing more towards the practice plan)?

Select ONE response for each option provided.

Parental leave

Elder-care leave

Child-care leave

Personal emergency leave

Family emergency leave (1st degree relative)

Bereavement Leave

Comments 
26. My .... hosts meetings at times that allow me to participate (i.e. during routine business hours for your division).

Select ONE response for each option provided.

Not Never or

Applicable Rarely Infrequently Sometimes Frequently Always

Hospital Division

Hospital DOM

University Division

University DOM

Research Institute

Comments

27. How frequently do you use the following patient care strategies in your daily practice? Select ONE response for each option provided.

Not Never or

Usually

E-consults through MOHLTC [1]

E-consults directly with colleagues

Email correspondence with patients

Telemedicine assessments of patients

[1] MOHLTC $=$ Ministry of Health and Long-Term Care 


\section{Faculty Survey}

Department of Medicine (DoM) Faculty Survey 2019

SECTION 4: COMMUNICATIONS

The DOM wants to reach its members in a way that is convenient and relevant. Please take a moment to answer these questions that will tell us how you want to hear from us and about the content of the communications.

\section{How often do you visit the DoM website?}

Select ONE response only.
Never or Rarely
Infrequently
Sometimes
Frequently
Usually or Always

29. In which form(s) do you prefer to receive information about the DoM? Select ALL responses that apply.

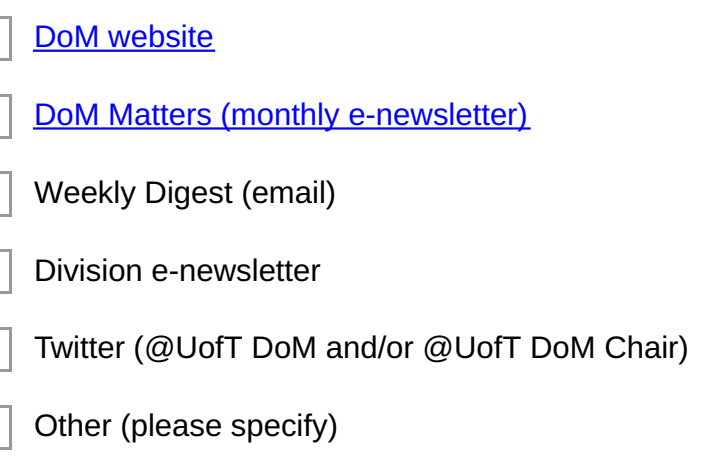


30. Please indicate your level of interest in each of the following topics:

Select ONE response for each option provided.

\section{Definitely}

not Slightly Moderately Very Extremely

Interested Interested Interested Interested Interested

Updates on DoM policies, procedures, strategic priorities

Faculty achievements

Trainee achievements

Alumni achievements

Upcoming deadlines (Funding opportunities, application

deadlines, nomination deadlines, etc.)

High-impact emerging research from our DOM

Upcoming events in all divisions, specialties and/or subspecialties

Other (please specify) 


\section{Faculty Survey}

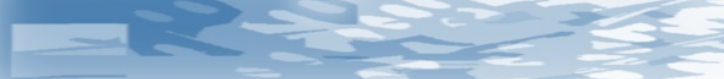

Department of Medicine (DoM) Faculty Survey 2019

\section{SECTION 5: QUALITY IMPROVEMENT}

The DoM wants to understand the impact of Quality Improvement on ALL faculty members, not just those in the Clinician in Quality and Innovation (C-QI) academic position description.

\section{In the past $\mathbf{1 2}$ months, have you participated in any of the following? Select ALL responses that apply.}
Led or collaborated on a QI [2] project or initiative primarily driven by academic interests
Led or collaborated on a QI [2] project or initiative at your clinical site primarily driven by operational priorities
$\square$ Led or participated in a patient safety event investigation (e.g., root cause analysis)
$\square$ Attended an educational activity (e.g., workshop, certificate course, rounds) with an explicit focus on quality/safety?
$\square$ Participated in QI [2] as part of creative professional activity for my academic position description
$\square$ None of the above

[2] QI = Quality Improvement

\section{Greater participation in QI activities by our faculty (not just by those in the CQI [3] job} description) has raised questions about the academic merit of QI. Which of the following statements best captures your perspectives about QI.

\section{Select ONE response only.}
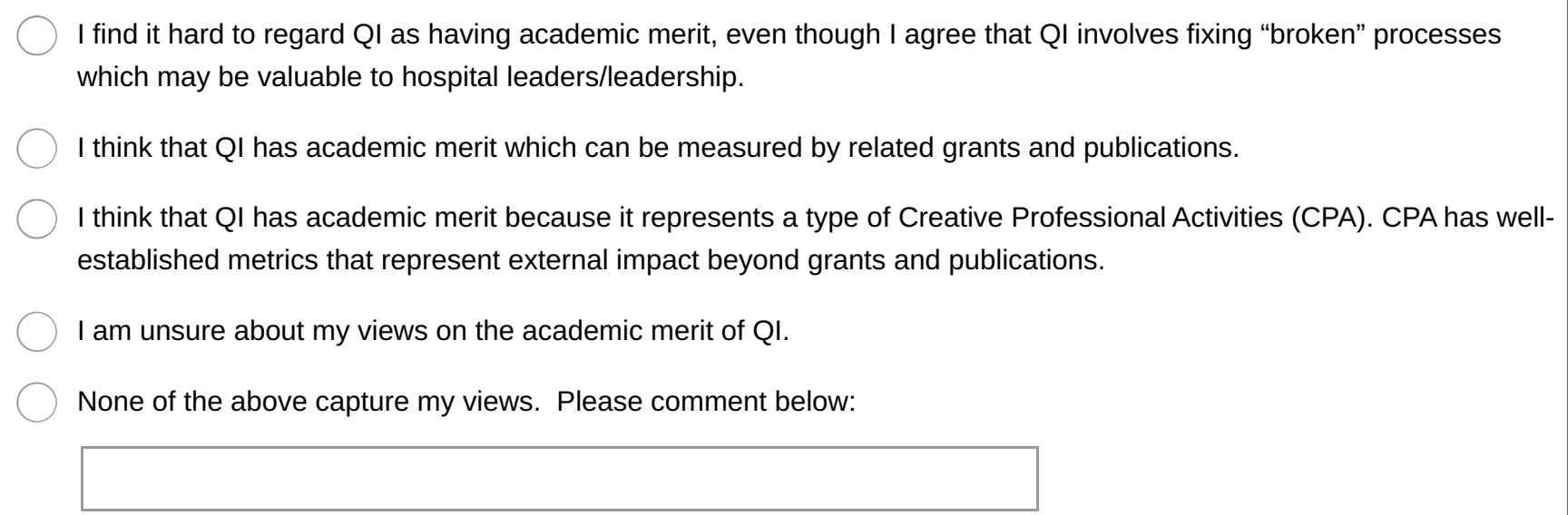

[3] CQI = Clinician in Quality and Innovation 


\section{Faculty Survey}

\section{Department of Medicine (DoM) Faculty Survey 2019}

\section{SECTION 5: DEMOGRAPHICS}

The DoM is collecting demographic information because of our commitment to diversity. We are tracking diversity in order to hold ourselves accountable and ensure we are reflecting the patient populations that we serve. Please note that the information you provide is anonymous and your privacy and confidentiality will be respected. The data you provide will not be linked with any other DoM databases and will only be analyzed in aggregate. Data will only be analyzed if there are $>=5$ respondents in a subgroup to protect the anonymity of respondents. You can answer none, some, or all of the demographic questions.

\section{Primary Hospital or Research Institute.}

Select ONE response only (the site you identify with most).

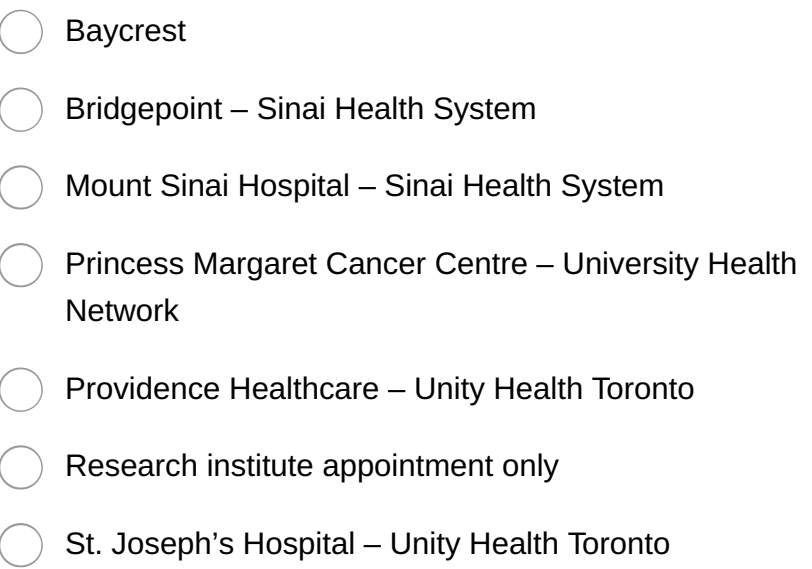
St. Michael's Hospital - Unity Health Toronto
Sunnybrook Health Sciences Centre
Toronto General Hospital - University Health Network
Toronto Western Hospital - University Health Network
Women's College Hospital
Prefer not to answer

\section{Select ONE response only.}

34. Division or Research Institute. Check your "primary" division.

Cardiology
Critical Care
Dermatology
Emergency Medicine
Endocrinology
Gastroenterology
General Internal Medicine
Geriatrics
Hematology
Infectious Diseases
Medical Oncology

Nephrology
Neurology
Physical Medicine and Rehabilitation
Respirology
Rheumatology
One of Allergy and Immunology, Clinical Pharmacology,
Pain, Occupational Medicine, Palliative Care
Other
Research institute appointment only
Not Applicable
Prefer not to answer


35. Academic Position Description

Select ONE response only.

Clinician Administrator (C-A)

Clinician Educator (C-E)

Clinician Investigator (C-I)

Clinician in Quality and Innovation (C-QI)

Clinician Scientist (C-S)

Clinician Teacher (C-T)

Research Scientist

Prefer not to answer

Other (please specify)

\section{Rank}

\section{Select ONE response only.}

Lecturer

Assistant Professor

Associate Professor

Full Professor

Emeritus (e.g. professor, dean, president)

Prefer not to answer

Other (please specify)

37. Age (years).

Select ONE response only.

$<=30$
$31-40$
$41-50$
$51-60$
$61-70$
$71-80$
$>80$
Prefer not to answer


38. What is your present gender identity?

Select ONE response only.

Woman

Man

Trans Woman

Trans Man

Gender Non-Conforming

Gender Fluid

Two-spirit

Prefer not to answer

Other (please specify):

39. What is your sexual orientation?

Select ONE response only.
Asexual/Non-sexual
Bisexual
Gay
Heterosexual ('straight')
Lesbian
Two-spirited
Pan-Sexual
Not sure/Questioning
Prefer not to answer
Other (please specify):

40. Do you care for any dependents (this may include a child, parent, other family member, relative with a disability, etc.)?

Select ONE response only.
Yes
No
Prefer not to answer

41. Do you consider yourself to be aperson of colour or member of a visible minorityin Canada? Select ONE response only.
Yes
No
Prefer not to answer 
42. Which of the following best describes your race/ethnic background?

Select ALL responses that apply.

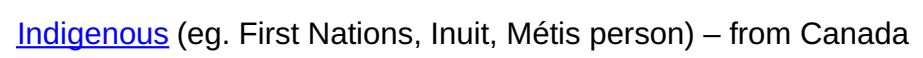

Indigenous - from another country

Black - African (eg. Ghanaian, Kenyan, Somalian, etc)

Black - North American (eg. Canadian, American) or Caribbean (eg. Barbadian, Jamaican, etc)

Latin / Hispanic (eg. Argentinian, Chilean, Salvadorian, etc)

East Asian (eg. Chinese, Japanese, Korean, etc.)

South Asian (eg. Indian, Pakistani, Sri Lankan, East Indian from Guyana, etc.)

Southeast Asian (eg. Filipino, Cambodian, Indonesian, Laotian, Vietnamese, Thai, etc.)

West Asian (eg. Iranian, Iraqi, Persian, etc.)

Central Asian (eg. Kazakh, Afghan, Tajik, Uzbek, Caucasus, etc.)

Middle Eastern

White - European (eg. British, Italian, Portugese, or Russian) or North American (eg. Canadian, American)

Mixed heritage

Prefer not to answer

Other (please specify)

43. Until the age of 16 , which of the following best describes your family's socio-economic status in the country you lived?

Select ONE response only.

Lower

Lower-middle

Middle

Upper-middle

Upper

Prefer not to answer

44. Whether or not it affects your day-to-day life, are you a person with adisability? Please check ONE only.
Yes
No
Not sure
Prefer not to answer 


\section{Faculty Survey}

\section{9}

Department of Medicine (DoM) Faculty Survey 2019

SECTION 5B: DEMOGRAPHICS I DISABILITY

45. Is your disability ...

Select ALL responses that apply.

Visible

Non-visible

Both

Prefer not to answer

\section{What type of disability do you have?} Select ALL responses that apply.

Physical, functional and/or mobility disability (e.g., arthritis, paraplegia, cerebral palsy, muscular dystrophy, spinal cord injuries, spina bifida)

Blind and/or low vision

Deaf, deafened and/or hard of hearing

Speech disability (e.g., stuttering)

Chronic medical condition (e.g., diabetes, chronic pain, HIVIAIDS, systemic exertion intolerance disease, kidney disease, seizure disorders)

Developmental disability (e.g., Asperger's Syndrome, Autism, Fetal Alcohol Spectrum Disorders)

Learning disability (e.g., dyslexia)

Psychiatric disability and/or mental health disability (e.g., bipolar disorder, obsessive compulsive disorder)

$\square$ Prefer not to answer

$\square$ Other (please specify) 


\section{Faculty Survey}

\section{9}

Department of Medicine (DoM) Faculty Survey 2019

FINAL COMMENTS

47. The DoM will be engaging in strategic planning this fall to lay out priorities for the next five years. Please take a moment to tell us what you think our DoM should be emphasizing as part of our next strategic plan:

48. If you could make one change in policy or practice that would improve your workplace as a place for all to work, what would it be?

49. Are there any missing items that should be included in future iterations of this survey?

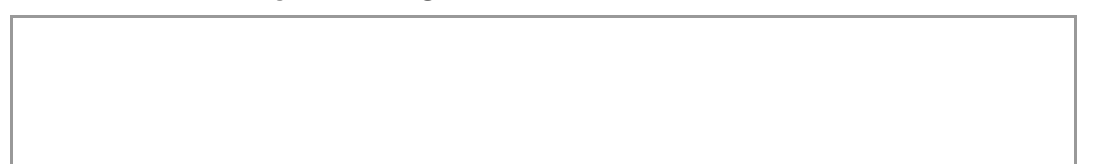

50. Are there any other comments or points on which you wish to elaborate?

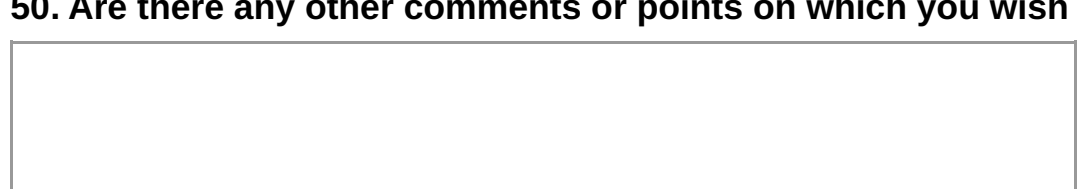




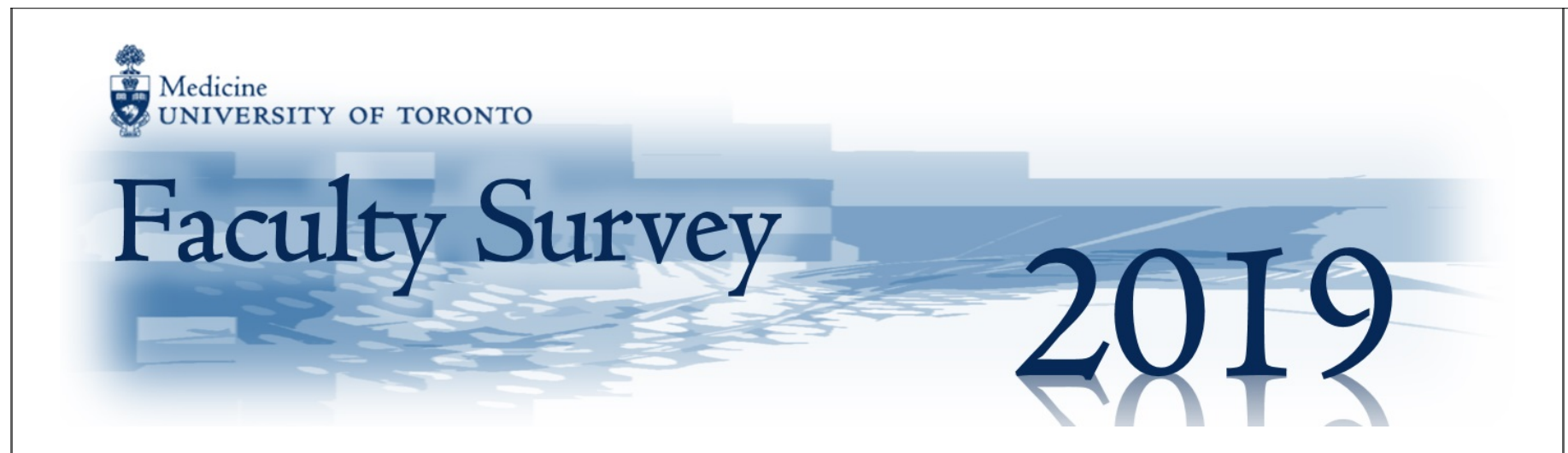

Department of Medicine (DoM) Faculty Survey 2019

\section{CONCLUSION}

Over the course of the survey, you may have identified some issues of concern. Here is a list of potential resources that we hope you will utilize if you need additional supports or guidance:

a. Clinical Faculty Advocate (CFA) within the Faculty of Medicine:

https://www.deptmedicine.utoronto.ca/clinical-faculty-advocate

b. Mentorship Facilitator: https://www.deptmedicine.utoronto.ca/mentorship-facilitators

c. OMA Wellness resources including Toll-free line:http://php.oma.org/wellnessResourcesYou.html

d. DoM Leadership, University of Toronto: https://www.deptmedicine.utoronto.ca/our-leadership 